\title{
Difficulty of cervical cancer diagnosis during pregnancy: A case series analysis of the clinicopathological characteristics and prognosis of cervical cancer diagnosed during pregnancy or within 6 months after parturition
}

\author{
KENZO SONODA $^{1,2}$, TATSUHIRO OHGAMI ${ }^{2}$, MASAHIRO HACHISUGA ${ }^{2}$, YASUYUKI FUJITA ${ }^{2}$, \\ KAORU OKUGAWA ${ }^{2}$, HIDEAKI YAHATA ${ }^{2}$ and KIYOKO KATO ${ }^{2}$ \\ ${ }^{1}$ Gynecology Service, National Hospital Organization Kyushu Cancer Center, Fukuoka 811-1395; ${ }^{2}$ Department of Obstetrics \\ and Gynecology, Graduate School of Medical Sciences and University Hospital, Fukuoka 812-8582, Japan
}

Received May 16, 2020; Accepted January 21, 2021

DOI: $10.3892 / \mathrm{mco} .2021 .2229$

\begin{abstract}
Due to the recent trend of women having children at an older age, the number of pregnancies complicated by cervical cancer has increased. In the present study, the clinical characteristics of patients with cervical cancer complicated by pregnancy were analyzed. The clinicopathological characteristics and prognosis of patients with cervical cancer during pregnancy who underwent treatment at Kyushu University Hospital from January 2008 to December 2017 were assessed retrospectively from their medical records. The medical information of patients diagnosed within 6 months after parturition was also evaluated as these patients were considered to be affected by cervical cancer during pregnancy. A total of 19 women were diagnosed with cervical cancer during pregnancy (median age, 33 years), three of whom were diagnosed as negative for intraepithelial lesion or malignancy at the initial visit to a previous clinic after pregnancy. The tumor stage was IA1 in one patient, IB1 in 16 patients, IB2 in one patient and IVB in one patient. The median gestational age at the time of cervical cancer diagnosis was 13 weeks. One patient died of cervical cancer during the follow-up period. An additional 12 patients were diagnosed within 6 months after parturition. The median age of these patients was 35.5 years. Cancer screening was performed in 10 patients during pregnancy, none of whom were diagnosed with cervical cancer, including three patients who were negative for intraepithelial lesion or malignancy. Of the 12 patients, one had tumor stage IA1, eight had IB1, two had IB2 and one had IIB. Three patients experienced recurrence, of whom one died of cervical
\end{abstract}

Correspondence to: Dr Kenzo Sonoda, Gynecology Service, National Hospital Organization Kyushu Cancer Center, Notame 3-1-1, Minami-ku, Fukuoka 811-1395, Japan

E-mail:kenzo@med.kyushu-u.ac.jp

Key words: uterine cervical neoplasms, pregnancy, parturition, diagnosis, prognosis cancer. Advanced cervical cancer was diagnosed both during pregnancy and within 6 months after parturition. Diagnosis of cervical cancer during pregnancy is difficult even at an advanced stage; however, rapid diagnosis and prompt multidisciplinary treatment are critical. Therefore, it is necessary to improve the accuracy of cervical cancer diagnosis, and to characterize tumor cells and their microenvironment, during pregnancy.

\section{Introduction}

The number of pregnancies complicated by cancer is increasing, and the incidence of any cancer development during pregnancy was recently estimated to be $1 / 1,000$ deliveries (1). According to the International Network on Cancer, Infertility and Pregnancy, breast cancer is the most common malignant tumor $(39 \%)$ in pregnant women, followed by cervical cancer (13\%), lymphoma (10\%), ovarian cancer (7\%), and leukemia (6\%) (2). Cervical cancer is the eighth most common malignancy in women and is especially prevalent in developing regions, with an estimated 569,847 new cases and 311,365 deaths reported in 2018 (3). In Japan, the incidence of cervical cancer has increased, from 11,053 cases diagnosed in 1975 to 34,120 cases in 2017 (4). The spread of human papillomavirus (HPV) has caused the incidence of precancerous and cancerous cervical lesions to increase, particularly in younger women of childbearing age (5). Cervical cancer has recently become the most common gynecological malignancy diagnosed during pregnancy (4/100,000 deliveries) (1). There have been several articles on treatment recommendations for cervical cancer in pregnant women. In addition, recommended treatments are briefly referred to in the National Comprehensive Cancer Network guidelines, version 1 (2021), for cervical cancer (6), and the Japan Society of Gynecologic Oncology published treatment guidelines in 2017 (7). However, treatment decision making is often difficult, and the decision to delay treatment until fetal maturity or initiate treatment immediately is made based on tumor stage. On the other hand, cervical cancer is often not diagnosed until shortly after delivery, because cytological and colposcopic findings change 
during pregnancy, decreasing diagnostic accuracy (8). The diagnosis and clinical course of cervical cancer complicated with pregnancy were assessed retrospectively in this study.

\section{Patients and methods}

Patients. We reviewed the records of all patients with cervical cancer during pregnancy or within 6 months after parturition who were treated at Kyushu University Hospital between January 2008 and December 2017. The patients had received no treatments prior to diagnosis. Patients were followed every 3 months during the first year after surgery, every 4 months during the second year, biannually during the third to fifth years, and annually thereafter (9). Follow-up included an extensive history review, physical and pelvic examinations, Papanicolaou smear, measurements of serum tumor markers, and imaging studies. Clinicopathological variables [patient age, FIGO (2008) tumor stage, histological subtype, tumor size, surgery type, and administration of adjuvant therapy] and data on recurrence were collected and analyzed retrospectively.

Cytological and histopathological examinations. Cytological specimens were collected from the cervix using cotton-tipped applicators. Because Japanese guidelines for gynecological practice published in 2011, 2014, and 2017 recommend using a cotton-tipped applicator for cervical cancer screening during pregnancy. Liquid-based cytology (LBC) was performed starting in April 2013. Ten and eight patients underwent LBC during pregnancy and within 6 months after parturition, respectively. A smear preparation method was applied for the remaining patients. The conventional Papanicolaou method was employed for cell staining, and the recommendations of the Bethesda System for Reporting Cervical Cytology, published in 2014, was used for diagnosis. In patients with suspicious or pathological cytology results, punch biopsy under colposcopy was conducted for histological evaluation. Neither HPV typing nor p16 immunocytochemistry was performed for cytological diagnosis in this study.

Ethics approval and consent to participate. The protocol for this study was approved by Kyushu University Hospital review board (authorization no. 622-00). All procedures were conducted in accordance with the ethical standards of the Institutional Research Committee of Kyushu University Hospital, the ethical guidelines of the Ministry of Health, Labour, and Welfare of Japan, and the 1964 Declaration of Helsinki and its later amendments. We only analyzed the data and medical or radiological images for which patient consent was obtained during the first consultation at our hospital. No patient-identifiable data were reported, and no direct interaction with the patient was necessary.

\section{Results}

Patients with cervical cancer during pregnancy. Of 815 patients with invasive cervical carcinoma who underwent treatment at our institute from January 2008 to December 2017, 19 were diagnosed during pregnancy (Tables I and SI). However, three of these patients (15\%) were diagnosed as negative for intraepithelial lesion or malignancy (NILM) at the initial visit to a previous clinic after pregnancy. The median age of these 19 patients was 33 (range, 25-45) years. The tumor stage was IA1 in 1 patient (5\%), IB1 in $16(85 \%)$, IB2 in $1(5 \%)$, and IVB in $1(5 \%)$. Ten patients (53\%) had squamous cell carcinoma, seven (37\%) had adenocarcinoma, and two (10\%) had adenosquamous cell carcinoma. The median gestational age at the time of cervical cancer diagnosis was 13 (range, 4-36) weeks. All but two patients (10\%) who underwent conization alone received radical therapy: After an abortion in 10 patients (53\%) (1 spontaneous and 9 artificial abortions), after artificial premature birth in 5 (27\%), and after full-term delivery in $2(10 \%)$. One stage IVB patient $(5 \%)$ died of cervical cancer during the follow-up period [median, 50 (range, 3-100) months]. No patient received chemotherapy during pregnancy. The patient with stage IA1 disease (patient no. 1) underwent conization at 18 weeks of gestation, and re-conization after full-term delivery showed no residual lesion. One patient with stage IB1 (no. 6) who delivered her baby vaginally did not experience recurrence, including at the episiotomy scar site. One patient (no. 15) requested trachelectomy during pregnancy and was thus transferred to another hospital; she underwent radical abdominal trachelectomy at 19 weeks of gestation and caesarean section and hysterectomy at 29 weeks of gestation.

Patients with cervical cancer within 6 months after parturition. An additional 12 patients were diagnosed within 6 months after parturition [median, 2 (range, 1-6) months after parturition] (Tables 1 and S1). The median age of these 12 patients was 35.5 (range, 27-40) years. Two patients (17\%) received no screening, and one was diagnosed with a cervical fibroma during pregnancy. Cancer screening was performed in 10 patients, of whom 3 were diagnosed as NILM (25\%), 2 (17\%) with atypical squamous cells of undetermined significance or low-grade squamous intraepithelial lesions, 1 (8\%) with cervical intraepithelial neoplasia (CIN) grade 2, and 4 (33\%) with CIN grade 3 . The tumor was stage IA1 in 1 patient (8\%), IB1 in $8(67 \%)$, IB2 in $2(17 \%)$, and IIB in 1 patient (8\%). Ten patients (84\%) had squamous cell carcinoma, $1(8 \%)$ had adenocarcinoma, and $1(8 \%)$ had adenosquamous cell carcinoma. Seven (58\%) and $2(17 \%)$ patients were diagnosed with cervical cancer after vaginal delivery and after caesarean section, respectively. Multidisciplinary treatment was conducted after diagnosis. Three patients (25\%) experienced recurrence (one patient each after vaginal delivery, caesarean section and spontaneous abortion) during the follow-up period [median, 60 (range, 27-77) months], of whom one stage IB2 patient died of cervical cancer. Two patients who had no evidence of disease after treating the recurrence underwent concurrent chemoradiation in the pelvis and para-aortic area.

Of the four patients who experienced recurrence (one with stage IVB diagnosed during pregnancy, and two with stage IB1 and one with stage IB2 diagnosed within 6 months after parturition), the one with stage IVB and the one with stage IB2 died. The clinicopathological variables and prognosis did not differ between the 19 and 12 patients diagnosed before and after delivery, respectively.

\section{Discussion}

In this study, the clinical characteristics of cervical cancer complicated with pregnancy were analyzed. The 


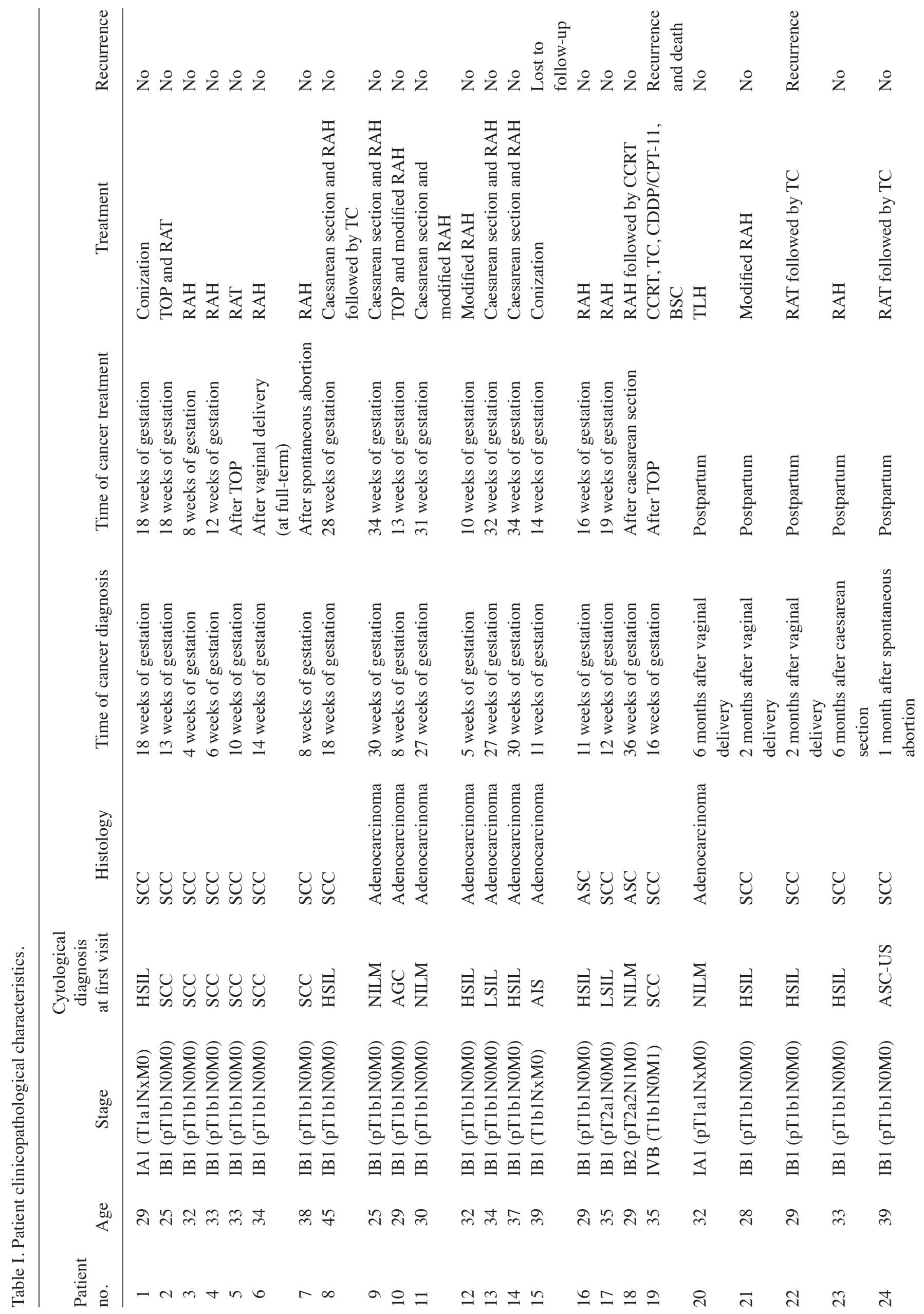




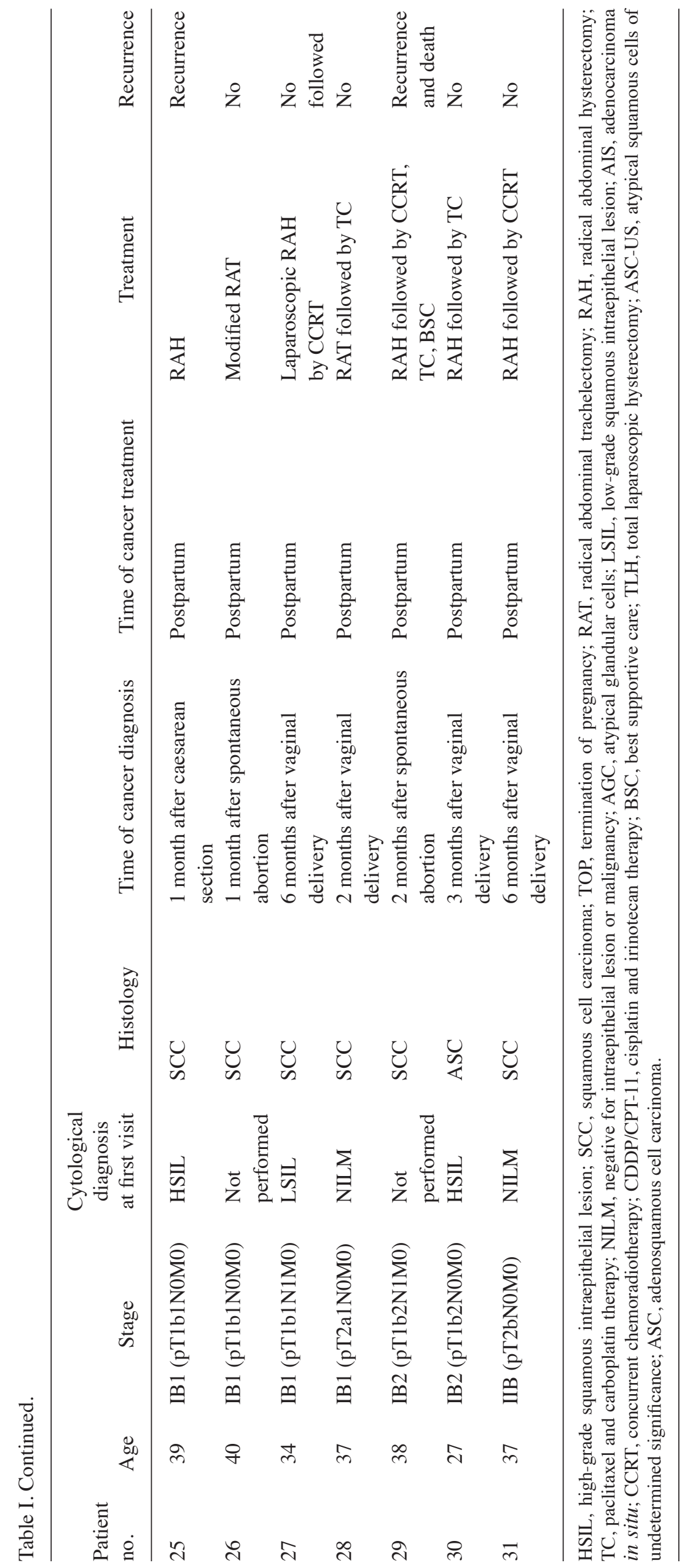


clinicopathological variables and prognosis of patients diagnosed with cervical cancer during pregnancy or within 6 months after parturition were evaluated. Because the latter patients were considered to be affected by cervical cancer during pregnancy. We experienced 31 cervical cancer patients who were diagnosed during pregnancy or within 6 months after parturition among 815 patients with invasive cervical carcinoma who underwent treatment at our institute from 2008 to 2017. Of these 31 patients, 19 were diagnosed with cervical cancer during pregnancy, although 3 of them were diagnosed as NILM at the initial visit to a previous clinic. An additional 12 patients were diagnosed with cervical cancer within 6 months after parturition, none of whom were diagnosed during pregnancy. Of the four patients who experienced recurrence, two who were diagnosed with advanced cervical cancer died of their disease.

Van Calsteren et al recommended visual inspection, cytological examination, and bimanual palpation as part of routine antenatal care, since pregnancy represents an exceptional opportunity for early diagnosis of cervical cancer (10). The rate of pregnant women presenting with abnormal cervical cytology has increased to approximately $5 \%$ of all pregnancies (11); approximately $50 \%$ of cervical cancer patients are diagnosed during pregnancy and the other 50\% during the first 12 months after delivery (12).

Treatment for cervical cancer is conducted at our institute in accordance with the Japan Society of Gynecologic Oncology guidelines (2017) for the treatment of uterine cervical cancer (7). On the other hand, Amant et al recommend different treatments depending on the gestational week and disease stage (13). Management without pregnancy preservation is recommended for advanced cases, and radical hysterectomy with the fetus in utero or post-hysterotomy is preferred for operable cases. Concurrent chemoradiation is applied for non-operable patients. Alternatively, cone biopsy can be performed for stage IA1 tumors without lymphovascular invasion, as a pregnancy-preserving treatment. For stage IA1 with lymphovascular invasion, IA2, and IB1 cervical cancer, staging lymphadenectomy is recommended and can be safely performed up to 22 weeks of gestation. The presence of lymph node metastasis necessitates termination of the pregnancy and standard treatment depending on the disease stage. In the absence of lymph node metastasis, trachelectomy or delayed treatment after delivery are considered for patients with stage IA2 or IB1 tumors smaller than $2 \mathrm{~cm}$ (14). Trachelectomy is a fertility-sparing surgery in which the cervix is resected and the uterine corpus preserved; however, radical trachelectomy is not strongly recommended during pregnancy because of a high rate of surgical and obstetrical complications, e.g., massive bleeding, severe intrauterine infections, and abortion (15). Of the 31 total patients, 8 who desired to bear children underwent fertility-sparing surgery (conization or trachelectomy) in our retrospective study.

Han et al evaluated the attitudes and knowledge of physicians regarding the treatment possibilities for patients with cancer during pregnancy (16). They found that termination of the pregnancy, delayed maternal treatment, and iatrogenic preterm delivery were the strategies most frequently used for management of pregnant cancer patients. Although the oncological prognosis is reportedly equivalent to that of non-pregnant patients, pregnant cancer patients should be treated in a multidisciplinary setting with access to maternal and neonatal intensive care units (17).

Diagnosis is often delayed during pregnancy because any bleeding that occurs is attributed to pregnancy-related complications, resulting in the development of advanced-stage cervical carcinoma that is not diagnosed until the antenatal or postpartum period. Papanicolaou test results during pregnancy may not be accurate because of the influence of pregnancy-associated hormones (18). In addition, colposcopic examination is difficult, and the diagnosis of 'atypical squamous cells, cannot exclude high-grade squamous intraepithelial lesion' was found to be less predictive of underlying high-grade squamous intraepithelial lesions in pregnant women compared with the general population (19). On the other hand, p16/Ki-67 dual-staining cytology may provide a valuable and novel approach to identify persistent or progressive CIN in pregnant women, and it may improve the management of abnormal Papanicolaou cytology results during pregnancy (20). Cytological specimens were collected using cotton-tipped applicators, and neither HPV typing nor p16 immunocytochemistry was performed for cytological diagnosis in this study. On the other hand, it has been reported that a nylon brush with a spatula can collect more cells without causing severe adverse effects to either the mother or fetus (21). The method of cell collection and the diagnostics of cytological samples should be reconsidered during pregnancy. In addition, patient counseling should address the issue that risk estimation may not be precise because of possible underestimation of disease severity during pregnancy.

Regarding cervical cancer diagnosed shortly after parturition, patient survival is reportedly worse and the risk of recurrent disease much higher, especially in the case of vaginal delivery, compared with patients diagnosed during pregnancy. According to a multivariate analysis in a previous study, vaginal delivery was the most significant predictor of recurrence, followed by advanced stage (22). Carocha et al reviewed cervical cancer patients with recurrent episiotomy and found that $80 \%$ of those with a vaginal delivery were diagnosed within the first 6 months postpartum (23). However, they recommended longer-term and more vigilant follow-up because of reports of metastasis from episiotomy scar sites being diagnosed 5 years after parturition. Of our patients who were diagnosed with cervical cancer within 6 months after parturition, seven and two were diagnosed after vaginal delivery and caesarean section, respectively. Three patients experienced recurrence (one patient each after vaginal delivery, caesarean section and spontaneous abortion), of whom one stage IB2 patient, who was diagnosed after spontaneous abortion, died of cervical cancer. No patients experienced recurrence at the site of the episiotomy scar.

A lowered immune response to human papilloma virus during the first two trimesters compared with the third trimester or postpartum period has been suggested (24). Although the rate of spontaneous regression of abnormal cervical cytology detected antepartum is higher after vaginal delivery than after caesarean delivery, frequent cytological and colposcopic evaluations seem safe. Due to the high rates 
of CIN grade 2 or 3 persistence during the postpartum period, Coppolillo et al recommended that all patients with CIN grade 2 or 3 diagnosed during pregnancy undergo biopsy and treatment if necessary during the postpartum period, with at least 2 years of follow-up to prevent lesion recurrence (25). Testing for high-risk HPV should be performed during follow-up of patients with squamous intraepithelial lesions detected during pregnancy or postpartum, in addition to cytology and colposcopy to detect persistence/progression of the lesions (26).

The current study had several limitations. First, only a small number of patients from a single institution were analyzed retrospectively. Therefore, the adverse effects of pregnancy on cervical cancer and prognosis were not sufficiently assessed. Second, the high heterogeneity in the clinicopathological variables, such as treatments and outcomes, among the patients limits explanation of our results. However, we evaluated the clinical characteristics of patients with cervical cancer diagnosed from the antepartum to early postpartum period. In conclusion, diagnosis of cervical cancer during pregnancy is difficult even at an advanced stage; however, rapid diagnosis and prompt multidisciplinary treatment are critical. Therefore, it is necessary to improve the accuracy of cervical cancer diagnosis, and to characterize tumor cells and their microenvironment, during pregnancy.

\section{Acknowledgments}

Not applicable.

\section{Funding}

No funding was received.

\section{Availability of data and materials}

All data generated or analyzed during the current study are included in this published article.

\section{Authors' contributions}

KS contributed to the data collection, analysis, and interpretation and the writing of the manuscript. TO, MH, YF, KO, HY, and KK contributed to the data analysis and interpretation and revision of the manuscript. KS and TO confirm the authenticity of all raw data. All authors contributed intellectually, and red and approved the final manuscript.

\section{Ethics approval and consent to participate}

The protocol for this study was approved by Kyushu University Hospital review board (authorization no. 622-00). All procedures were conducted in accordance with the ethical standards of the Institutional Research Committee of Kyushu University Hospital, the ethical guidelines of the Ministry of Health, Labour, and Welfare of Japan, and the 1964 Declaration of Helsinki and its later amendments. We only analyzed the data and medical or radiological images for which patient consent was obtained during the first consultation at our hospital. No patient-identifiable data were reported, and no direct interaction with the patient was necessary.

\section{Patient consent for publication}

All patients provided consent to use their personal data for scientific purposes.

\section{Competing interests}

The authors declare no conflicts of interest.

\section{References}

1. Hecking T, Abramian A, Domröse C, Engeln T, Thiesler T, Leutner C, Gembruch U, Keyver-Paik MD, Kuhn W and Kübler K: Individual management of cervical cancer in pregnancy. Arch Gynecol Obstet 293: 931-939, 2016.

2. de Haan J, Verheecke M, Van Calsteren K, Van Calster B, Shmakov RG, Mhallem Gziri M, Halaska MJ, Fruscio R, Lok CAR, Boere IA, et al: Oncological management and obstetric and neonatal outcomes for women diagnosed with cancer during pregnancy: A 20-year international cohort study of 1170 patients. Lancet Oncol 19: 337-346, 2018.

3. World Health Organization/International Agency for Research on Cancer. Cervix uteri. Source: Globocan 2018. Available from: http://gco.iarc.fr/today/data/factsheets/cancers/23-Cervix-uteri-f act-sheet.pdf. Accessed October 31, 2020.

4. Cancer Registry and Statistics. Cancer Information Service, National Cancer Center, Japan (Monitoring of Cancer Incidence in Japan (MCIJ)). Cancer incidence. Available from: https://ganjoho.jp/en/professional/statistics/table_download. html. Accessed October 31, 2020.

5. Lynge E, Rygaard C, Baillet MV, Dugué PA, Sander BB, Bonde J and Rebolj M: Cervical cancer screening at crossroads. APMIS 122: 667-673, 2014.

6. National Comprehensive Cancer Network. Guidelines version 1.2021 for cervical cancer. Available from: https://www.ncen. org/professionals/physician_gls/default.aspx. Accessed October 31, 2020.

7. Ebina Y, Mikami M, Nagase S, Tabata T, Kaneuchi M, Tashiro H, Mandai M, Enomoto T, Kobayashi Y, Katabuchi H, et al: Japan society of gynecologic oncology guidelines 2017 for the treatment of uterine cervical cancer. Int J Clin Oncol 24: 1-19, 2019.

8. Origoni M, Salvatore S, Perino A, Cucinella G and Candiani M: Cervical intraepithelial neoplasia (CIN) in pregnancy: The state of the art. Eur Rev Med Pharmacol Sci 18: 851-860, 2014.

9. Sonoda K, Kobayashi H and Wake N: Cervical carcinoma surveillance counterpoint. In: Patient Surveillance After Cancer Treatment. Johnson FE, Maehara Y, Browman GP, Margenthaler JA, Audisio RA, Thompson JF, Johnson DY, Earle CC and Virgo KS (eds). Springer Science Business Media, New York, NY, pp335-340, 2013.

10. Van Calsteren K, Vergote I and Amant F: Cervical neoplasia during pregnancy: Diagnosis, management and prognosis. Best Pract Res Clin Obstet Gynaecol 19: 611-630, 2005.

11. Palle C, Bangsbøll S and Andreasson B: Cervical intraepithelial neoplasia in pregnancy. Acta Obstet Gynecol Scand 79: 306-310, 2000.

12. Smith LH, Dalrymple JL, Leiserowitz GS, Danielsen B and Gilbert WM: Obstetrical deliveries associated with maternal malignancy in California, 1992 through 1997. Am J Obstet Gynecol 184: 1504-1513, 2001.

13. Amant F, Berveiller P, Boere IA, Cardonick E, Fruscio R, Fumagalli M, Halaska MJ, Hasenburg A, Johansson ALV, Lambertini M, et al: Gynecologic cancers in pregnancy: Guidelines based on a third international consensus meeting. Ann Oncol 30: 1601-1612, 2019.

14. Morice P, Uzan C, Gouy S, Verschraegen C and Haie-Meder C: Gynaecological cancers in pregnancy. Lancet 379: 558-569, 2012.

15. Douligeris A, Prodromidou A, Psomiadou V, Iavazzo C and Vorgias G: Abdominal radical trachelectomy during pregnancy: A systematic review of the literature. J Gynecol Obstet Hum Reprod 49: 101607, 2020.

16. Han SN, Kesic VI, Van Calsteren K, Petkovic S and Amant F; ESGO 'Cancer in Pregnancy' Task Force: Cancer in pregnancy: A survey of current clinical practice. Eur J Obstet Gynecol Reprod Biol 167: 18-23, 2013. 
17. Van Calsteren K, Heyns L, De Smet F, Van Eycken L, Gziri MM, Van Gemert W, Halaska M, Vergote I, Ottevanger N and Amant F: Cancer during pregnancy: An analysis of 215 patients emphasizing the obstetrical and the neonatal outcomes. J Clin Oncol 28: 683-689, 2010.

18. Suzuki K, Furuhashi M, Kawamura T, Kubo M, Osato K and Yamawaki T: Comparing papanicolaou test results obtained during pregnancy and post-partum. J Obstet Gynaecol Res 43: 705-709, 2017.

19. Onuma K, Saad RS, Kanbour-Shakir A, Kanbour AI and Dabbs DJ: Clinical implications of the diagnosis 'atypical squamous cells, cannot exclude high-grade squamous intraepithelial lesion' in pregnant women. Cancer 108: 282-287, 2006.

20. Trutnovsky G, Kolovetsiou-Kreiner V and Reich O: p16/Ki-67 dual-stained cytology testing may predict postpartum outcome in patients with abnormal papanicolaou cytology during pregnancy. Acta Cytol 58: 293-296, 2014.

21. Holt J, Stiltner L, Jamieson B and Fashner J: Clinical inquiries. Should a nylon brush be used for Pap smears from pregnant women? J Fam Pract 54: 463-464, 2005.

22. Sood AK, Sorosky JI, Mayr N, Anderson B, Buller RE and Niebyl J: Cervical cancer diagnosed shortly after pregnancy: Prognostic variables and delivery routes. Obstet Gynecol 95: 832-838, 2000
23. Carocha AI, Pedroso C, Correia L, Gomes A and Jorge AF: Glassy cell carcinoma of the cervix and metastasis in episiotomy scar: A case report. J Low Genit Tract Dis 19: e31-e34, 2015.

24. Nobbenhuis MA, Helmerhorst TJ, van den Brule AJ, Rozendaal L, Bezemer PD, Voorhorst FJ and Meijer CJ: High-risk human papillomavirus clearance in pregnant women: Trends for lower clearance during pregnancy with a catch-up postpartum. Br J Cancer 87: 75-80, 2002.

25. Coppolillo EF, DE Ruda Vega HM, Brizuela J, Eliseth MC, Barata A and Perazzi BE: High-grade cervical neoplasia during pregnancy: Diagnosis, management and postpartum findings. Acta Obstet Gynecol Scand 92: 293-297, 2013.

26. Frega A, Scirpa P, Corosu R, Verrico M, Scarciglia ML, Primieri MR, Palazzo A, Iacovelli R and Moscarini M: Clinical management and follow-up of squamous intraepithelial cervical lesions during pregnancy and postpartum. Anticancer Res 27: 2743-2746, 2007.

(i) $($ This work is licensed under a Creative Commons Attribution-NonCommercial-NoDerivatives 4.0 International (CC BY-NC-ND 4.0) License. 